\title{
Learning path based-portfolio for personalized learning
}

\author{
Lucila Romero \\ GIDIS Research Group, \\ Facultad de Ingeniería y Ciencias Hídricas, \\ Universidad Nacional del Litoral, Argentina \\ lucila.rb@gmail.com
}

\author{
Milagros Gutierrez \\ CIDISI Research Center UTN, \\ Facultad Regional Santa Fe, Argentina \\ mmgutier@frsf.utn.edu.ar
}

\author{
Laura Caliusco \\ CIDISI Research Center UTN, \\ Facultad Regional Santa Fe, Argentina \\ mcaliusc@frsf.utn.edu.ar
}

\begin{abstract}
Nowadays, lot of human activities have been affected by the use of technology needed to change in order to adapted. Particularly, in education domain the changes that occurred were not enough to break the traditional schemas of teaching. In this way, more and more people claim for real remodeling. Mainly, one of the most important subject is the necessity to adapt the teaching process to the particular characteristics of students, in a king of personalized learning. This work presents a conceptual framework, which proposes learning path based-portfolio as a way to personalize education. This framework uses semantic technologies, logical rules and agents to determine the student learning level and based on that propose course of actions.
\end{abstract}

Keywords - learning path; portfolio; e-learning; ontologies; assessment.

\section{INTRODUCTION}

In the last decade, the use of technologies, particularly digital technologies in education (e-learning) has been growing, in that way nowadays, nobody can disagree with the importance and impact that it has in the modern life. However, like every tool, the student success will depend on the suitable use of it. The more the student is encouraged and engaging, the more probability the student success. One of the most important issue to take into account in order to keep student encouraged is the assessment. It is the main artifact used by teacher to keep in touch with the student and in this way, student feels observed [1]. Also, when teachers assess students, they can realize if students are reaching the various learning levels [13]. Then, they can propose new activities and assessment that help student to keep in-depth education. It is important to note that students learn at their own pace, this fact force to adapt the mechanism according with particular characteristics of students. Technologies can help teacher to discover who is learning and who is demotivated and proposes action, which can be for instance, when, whom and how assess, get new activities or stimulate to re-do the giving activities.

This work takes into account two possible situations in which student can be demotivated: (i) when student fail repeatedly to reach a level and (ii) when student reach the level quickly and them get bored. Both cases have to be taking into account to propose a solution in personalized learning. This proposal uses semantic web technology and artificial intelligence mechanism given a conceptual framework to the development of tools for personalized learning through portfolio definition, calculation of index, knowledge level identification and learning path recommendation. Portfolio is used to gather student experiences and his/her interaction with teachers. The knowledge level and index are used to infer action path recommendation. Learning paths are used to define a set of one or more learning activities leading to a particular learning goal.

In previous work, author have proposed an assessment model in e-learning environment [2-6], then they added the concept of portfolio as the container of assessment and student tracking $[8,9]$. This work is an improvement of previous one tackling the problem of environment adaptation according with the knowledge level that student has reached proposing a learning path.

This work is organized as follow. Section two presents the background knowledge used to develop this work. Section 2 presents the background knowledge in which this work is based. Section 3 shows the both indicators used to define learning path based-portfolio and the implementation through rules and set used by an agent to propose actions. Finally the work are concluded.

\section{BACKGROUND}

\section{A. Learning level}

A course is designed according with some objectives to achieve for each learning level, skills and behavior. Based on these, it is defined with lectures the course will have and in which level the student need to reach each one. The instructor plans each class and selects the appropriate methodology, the lectures, assignments and assessment in accordance with goals [10].

Teacher gives students lectures, assignment, projects and assessment according with the students' progress. In this way, if they success in reach a learning level, then they can follow to the next step in the educational process and access to the course materials belonging to the next learning level.

Here it is considered three learning levels, which each level is defined as the knowledge qualitative stage $[10,13]$ : (i) first learning level: know, make sense, understand something. At this level, students keep concepts in mind and can reproduce the information. But they cannot reason or exercise knowledge appropriately. (ii) second learning level: cognitive learning, deep learning. This level involves mental processes in learning. Students at this level can observe, categorize and forming generalization to make sense of the information provided. The learning results come from mental activities but not from externals stimulus. Learners gain knowledge, skills and experiences to apply in both real life and to solve problems. (iii) Third learning level: Create, evaluate, modify the already knowledge to take into account new information. Students at this level can justify a decision or course of action, generate new ideas, design new thing, construct, plan, make hypothesis, among others.

In order to achieve each learning level, it is necessary different learning activities such as expositive class, lecture, 
exercises, video, essay, assessment, case study, among others.

Learning is a spiral process by nature. Some time it requires frequent opportunities for reviewing learn material. Teachers have to be aware of which level their student have to reach in order to use the correct activities. If a professor every time uses expositive class, he/she only works for reaching the first learning level, and it is not enough to reach upper levels. Then, a professor has to assess students constantly in order to determine if student has reached a certain level, in which case he/she has to propose new activities for reaching the next level in the hierarchy. As regards assessment, it is important that has open question, because this type of reactive triggers mental processes, given as result a meaningful, permanent and effective learning [11].

Teachers can determine the students' learning level based on some index. For instance, they need to decide if students can discover new relationships and apply certainty and successfully acquired knowledge in practical activities. Nowadays they use score as the main index in order to measure the learning results, with the disadvantage of these scores are not homogeneous, depending on teachers' judgment, rigor and experiences. At that time, score are considered as weak indicators, and it is necessary to complement with another index and metrics [12].

The international educator community agree on some indicators to measure the learning results such as: (i) rate of correct answer, (ii) rate of correct answer in each level and (iii) rate of students that reach a learning level [13].

Sometime teachers fail in guide the student to reach the third level, when they propose to reproduce knowledge learned by heard and in some circumstances reproducing irrelevant data.

If teacher use scientific information in the third level in a correct way, they will guarantee a complete and legitimate learning coherent with society hope and institutional goals.

\section{B. Portfolios}

Nowadays portfolio is increasingly playing a leader role in the educational context, particularly at university with different purposes such as the use of portfolios as tool to plan individual course of action depending on student skill and capacities.

E-portfolio is the name used for defining portfolios in e-learning environment as well as portfolio assessment is the name used for defining portfolios use to assess students [15].

E-portfolio is a set of digital artifacts including proof, comments, resources, answers, among others. It can contain lectures, assignment, assessment, teacher comment, activities, and other artifacts that teacher give student in the course [16].

One of the objective to use e-portfolios is assess student in a personalized way, taking into account individual characteristics and the student pace of learning [17]. In this way, assessment has its electronical version into the e-learning environment [18]. Further e-portfolios can contain type of assessment tools in order to measure different learning level such as objective assessment, essay, and conceptual maps, among others. From the point of student view, e-portfolio provides both an interactive and intimate way of keeping in touch with teachers and thoughtful evidences with different e-assessments [19].

Each student organizes its own e-portfolio as best as possible for him/her taking into account the teacher's advice. So, e-portfolio encourages the self-organized learning and development a reflexive thought. For that, teachers need to set some rule in order to guide students in this task. For instance, teachers can propose different type of assessment such as hetero assessment, self-assessment and co-assessment [20]. Then the student's answer associated with these assessments will be available to teacher with the aim of both teacher measures the learning level and student be able to self-organize activities. At the same time, teacher can have its own portfolio where collect learning objects (LO) for offering to the student.

In recent years, it has been developed large number of e-portfolios systems as observed in [21]. They offering to teacher and student the possibility to generate its own portfolios, personalize it and keep track of their progress. These systems use different technologies and are integrated with learning management system (LMS) with lot of functionalities.

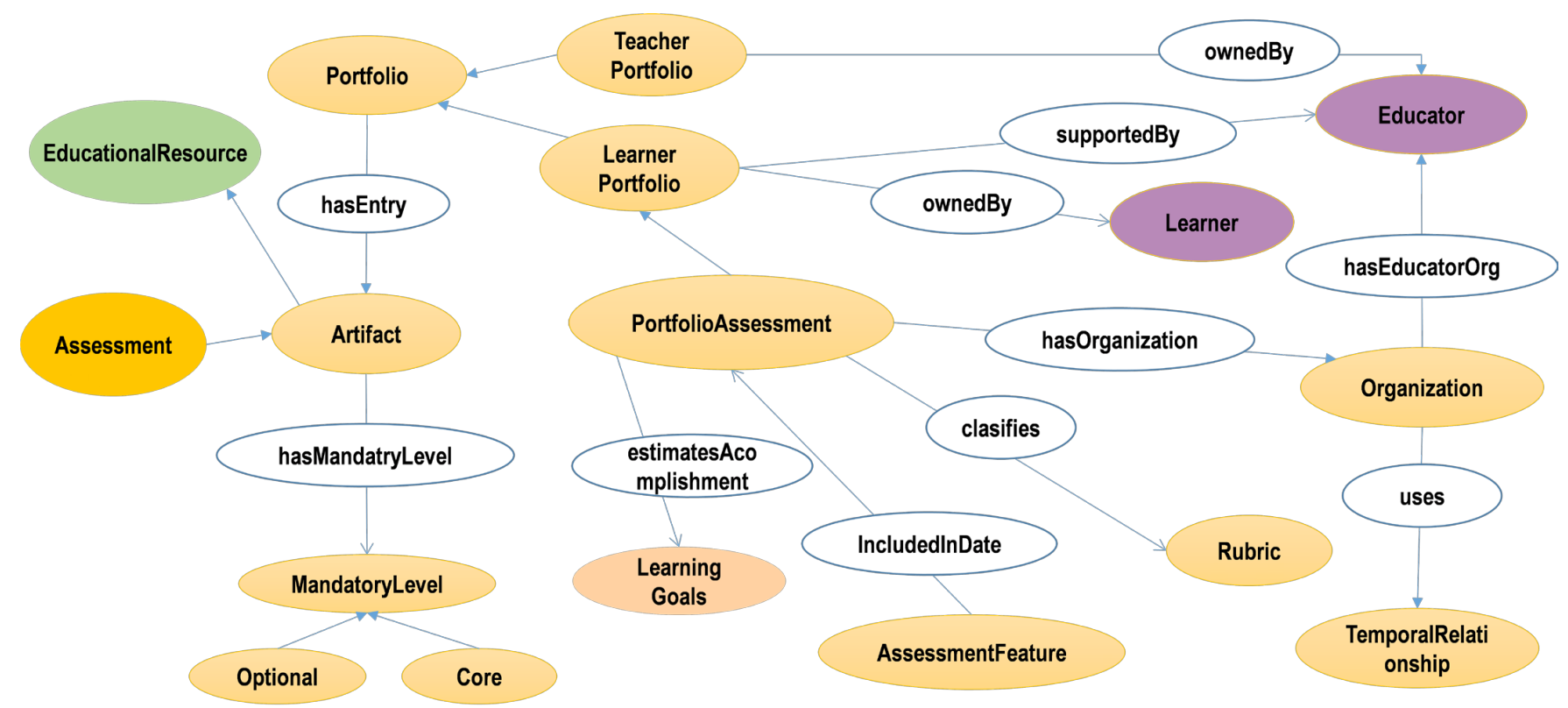

Figure 1: ePortfolios Ontology 
III. MODEL OF ASSIMILATION INDICATORS BASED ON SEMANTIC TECHNOLOGIES

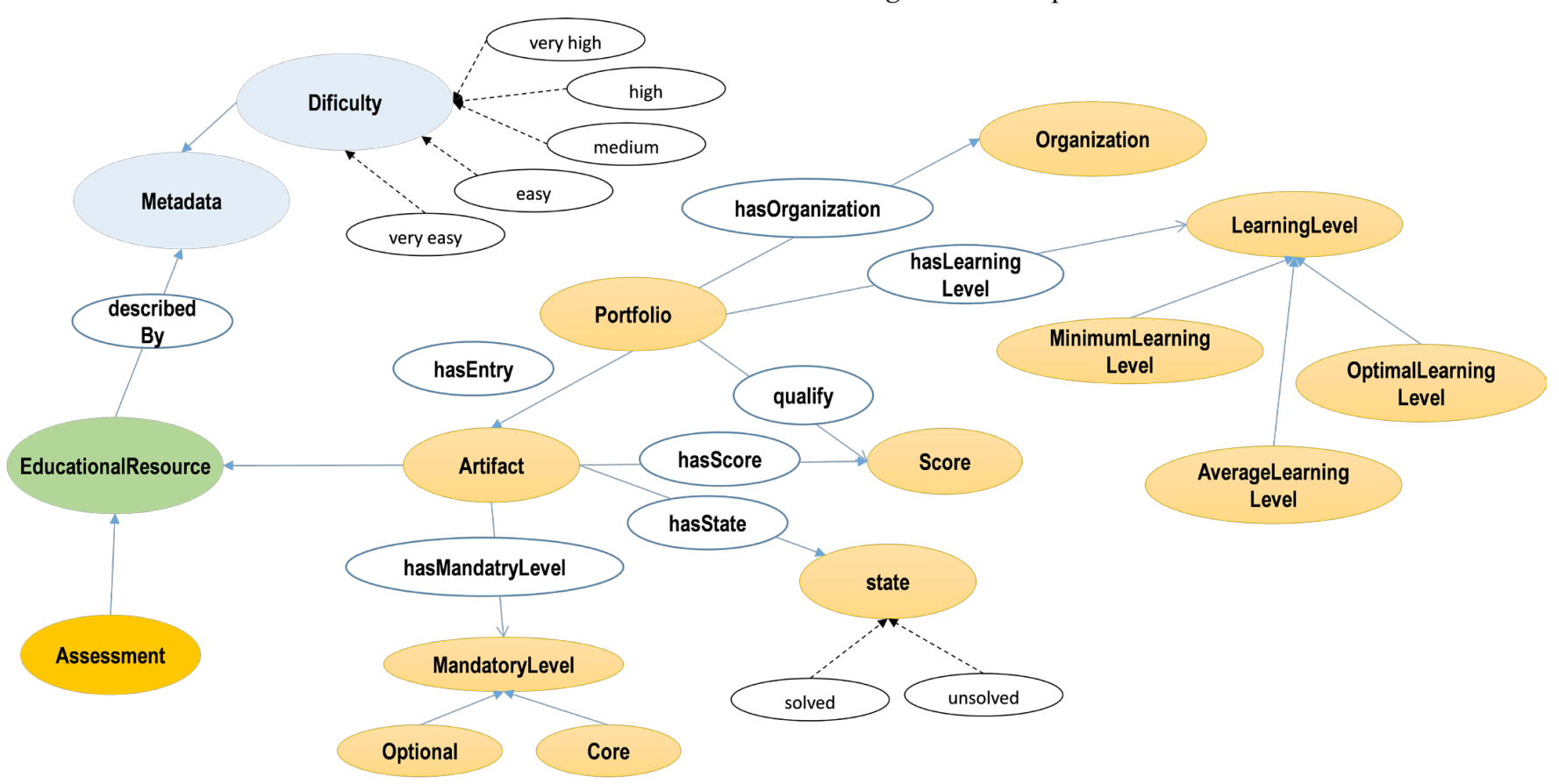

evaluations among others. It is clear that the LearningLevel and LearningGoals concepts are related to one another. The value

Figure 2: Indicators' Terms and values

This work is the continuation of previous works that have been carried out in the area, where a network of ontologies called AONet was defined for the semiautomatic generation of assessment in e-learning context $[6,7,8,9]$. The e-portfolio ontology that conceptualizes portfolios shown in figure 1 is part of the network.

Following this process, this work presents a new iteration containing other domains and extended ontologies.

\section{A. Indicators Definition}

This work defines some indicators that allow teacher both to determine the learning level reached by students and to direct and personalize the learning process making decisions about the most appropriate materials to use for this purpose.

So, the e-portfolio ontology has been enrich adding new concepts that refer to measures and new metadata to better describe the LO that are part of the portfolio. Authors decided to consider the portfolio (Portfolio in the ontology) since it represents a set of LO used to determine the student's learning level.

Figure 2 shows concepts and values for each one use to calculate indicators. The LearningLevel concept refers to the learning level and has three sub-concepts: OptimalLearningLevel, AverageLearningLevel and MinimumLearningLevel, representing the optimal, average and minimum learning levels respectively. Teacher has to specify numerical values for each level prior to the progress of learning on a given topic according with some criterion. Then, learning level will be associated with a portfolio. The numerical values represent the score that the student has to be achieve in assessment.

LearningLevel relates to the PortfolioAssessment concept through the hasLearningLevel relationship, expressing that a portfolio of assessments has an associated learning level. The concept LearningLevel relates to the concept Educator through the concept establishes expressing that a teacher establishes the different learning levels of the portfolio according to their content: materials, work done, proposed self-assessments, of learningLevel will depend on the objectives established for each concept taught in course. In this sense, the relationship that links both concepts is depends. In the ontology, the portfolio qualification is modeled through the Score concept, which represents the student's portfolio score at a point in the learning process. This value should reflect the student results in resolving practical work, problem, essay, assessment, among others. This qualification relates to the concept Learner through the get relation meaning that the score is obtained by learner.

Portfolio concept is also related to Educator concept through the assign relationship meaning that is the teacher who assigns the qualification to the portfolio after evaluating its content when completing a stage of the path of the learning process. The Portfolio concept is related to the Score concept through the qualify relation, expressing that a portfolio has a score with a value assigned by teacher. The Score concept is also used for the grades obtained in the assessments. In this case the concept is related to the term Assessment through the hasScore relation expressing that an evaluation solved by a student has a grade after being corrected by the teacher.

After defining the main concepts needed to know about the learning level of a student, authors began working on possible indicators that provide quantitative information on the progress of learning. It is important to highlight that the purpose of the definition of the indicators is to propose recommendations to the professor through an application. This recommendation should be supplemented by the expert qualitative view of the professor. Indicators defined among others

For the systematization of these indicators, the teacher of the course is asked to define a series of values for some concepts used for their calculation. The concepts are shown in Figure 2. The LearningLevel refers to the learning level and has associated three subconcepts: OptimalLearningLevel, AverageLearningLevel and MinimumLearningLevel, which represent the three level consider in this work. These values must be specified by the teacher prior to the progress of teaching in a given subject. They are identified with a numerical value 
associated to a portfolio and represent the values to be achieved by the students that will determine the level of learning achieved.

LearningLevel se relaciona con el concepto PortfolioAssessment a través de la relación hasLearningLevel, expresando que un portfolio de evaluaciones tiene un nivel de aprendizaje asociado. El concepto LearningLevel se relaciona con el concepto Educator a través del concepto establishes expresando que un profesor establece los diferentes niveles de aprendizaje del portfolio de acuerdo a su contenido: materiales, trabajos realizados, autoevaluaciones propuestas, evaluaciones realizadas, etc. Es evidente que el concepto LearningLevel se relaciona con el concepto LearningGoals dado que el nivel de aprendizaje depende de los objetivos de aprendizaje establecidos para los conceptos impartidos en la asignatura. La relación que vincula ambos conceptos es la relación depends.

\section{B. Learning path Definition:}

In the last period great attention is paid in the introduction of methodologies and techniques for the adaptation of learning process to the real needs of students. In this paper, it is proposed to define a learning path taking into account the student progress in his/her learning process.

Learning paths are defined as sets of one or more learning actions that lead to a particular learning goal. These learning actions can be formal, non-formal, informal or a combination of these, and can vary from a relatively small activity, like reading a book, to following an entire curriculum [22].

Portfolio's qualification (PQ) is considered and compared to the learning levels associated with the portfolio at a given stage in the learning process. The calculation involves Difficulty metadata [14] whose possible values are very easy, easy, medium, high and very high. If PQ obtained by a learner is equal to the optimal level of the portfolio, continuity in learning process is suggested by making available for learner the material more complex. That is, materials, notes, practical assignments and self-assessments with Difficulty metadata value with high or very high values will be available to the student. On the other hand, if PQ is lower than the optimal level but equal to or higher than the average learning level in the portfolio, it will be suggested to continue with the next stage of learning process with medium difficulty materials (Difficulty metadata with medium value). Achieving this level means that the student has the minimum conditions to continue his study with the next step of the course. In another case, it means that learner can only reproduce concepts without being able to apply them or expose them in his own words, so it will be suggested to stay in the same stage of the program with materials and self-assessments of low or very low difficulty. Every LO, found in the portfolio, are modeled in the Artifact concept which is a subconcept of EducationalResource concept. EducationalResource concept has Difficulty metadata concept associated with the hasDifficulty relationship.

\section{Rules}

Table 1 shows the logical rules defined in the ontology to define in Section A, as well as the rules used by an agent in charge of proposing learning path in the application based on this ontology. In this case, the rules that are observed in the table identify the level reached by the student based on the qualification obtained so far in the portfolio (Score concept).

By applying these rules, the ontology identifies the learning level through predicates used: AnswerGoForware, AnswerGoAverage and AnswerGoMinimum. Also defined are the sets HIGH, AVERAGE and MINIMUM that contain all the LOs that are in a portfolio and whose levels of difficulty are high or very high for the first set; Medium for the second set and easy or very easy for the last of these sets. Then, it is possible to define a rule-based agent that recommends performing the LOs found in these sets defined according to the degree reached by the student.

- Design of an assessment: This indicator is intended to assist in the selection of activities or questions for an assessment of the portfolio. This indicator also takes into account the

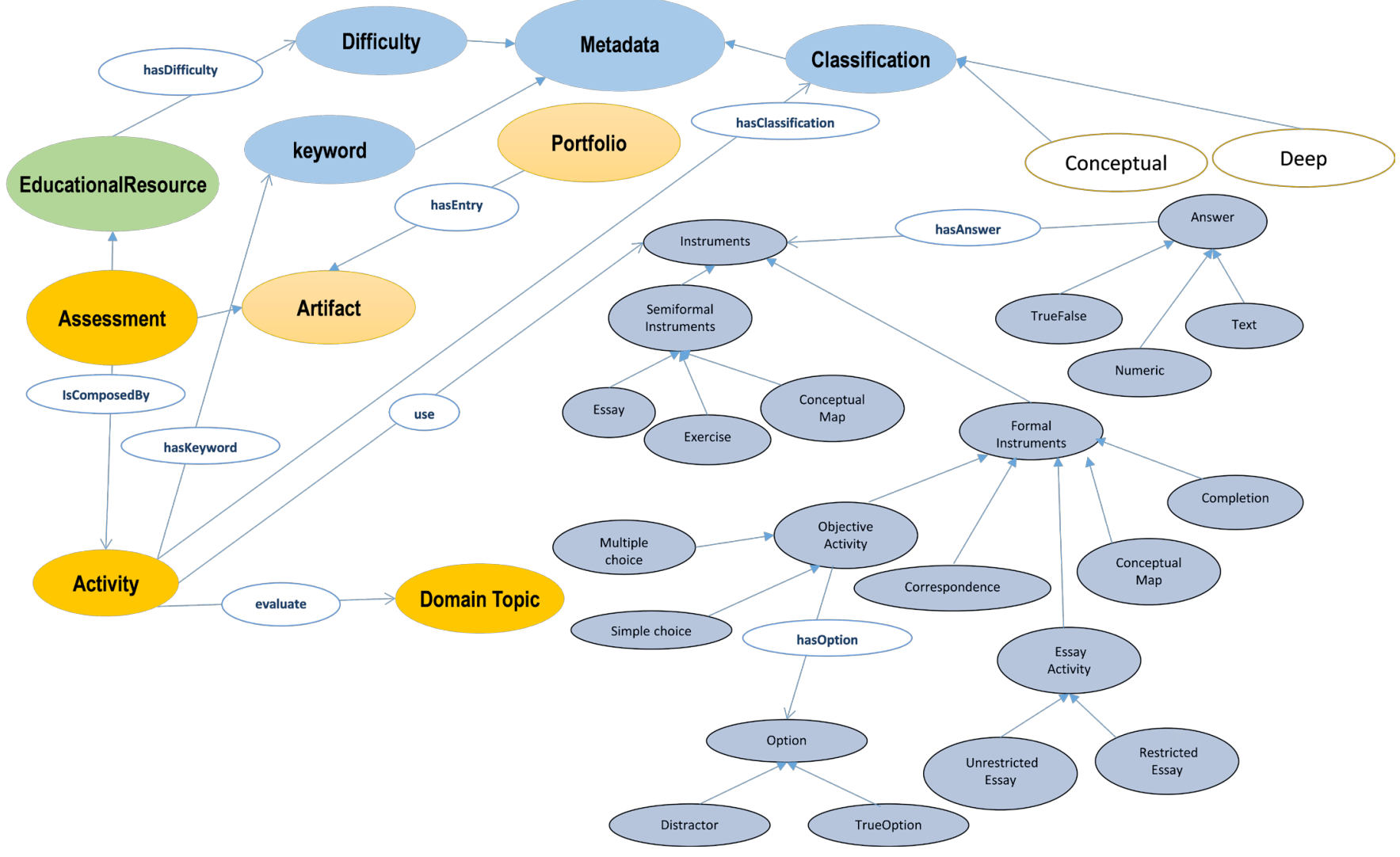

Figura 3: Ontologías de eportfolios. Clasificación de instrumentos 


\begin{tabular}{|c|c|}
\hline Description & Rule \\
\hline $\begin{array}{l}\text { If Portfolio qualification is higher than the set value in } \\
\text { OptimalLearningLevel then recommend Continue }\end{array}$ & $\begin{array}{c}\text { Portfolio(?p)^ }{ }^{\wedge} \text { qualify }(? \mathrm{p}, ? \mathrm{~s})^{\wedge} \text { hasLearningLevel(?p,?11)^} \\
\text { OptimalLearningLevel(?11) })^{\wedge} \text { sqwrl:greaterThanOrEqual(?s,?11) } \rightarrow> \\
\text { AnswerGoForward(?p) }\end{array}$ \\
\hline $\begin{array}{l}\text { If Portfolio qualification is higher than the set value in } \\
\text { AverageLearningLevel but lower than optimal then recommend } \\
\text { Advance with Media Difficulty }\end{array}$ & 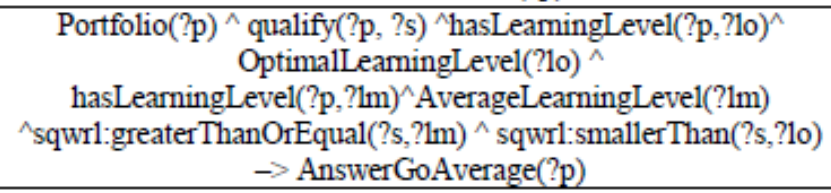 \\
\hline $\begin{array}{l}\text { If portfolio qualification is higher than the value set in } \\
\text { MinimunLearningLevel but lower than the AverageLeamingLevel } \\
\text { then recommend advancing with Minimum Difficulty. }\end{array}$ & 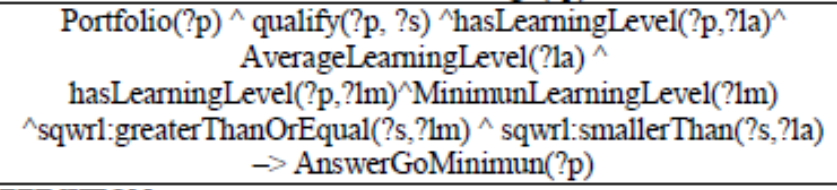 \\
\hline \multicolumn{2}{|r|}{ SET DEFINITION } \\
\hline $\begin{array}{l}\text { The HIGH set of all Learning Objects that are in the portfolio whose } \\
\text { difficulty is high and very high is defined }\end{array}$ & $\begin{array}{c}\text { HIGH } \equiv \text { Artifact } \sqcap \exists \text { describedBy. }(\text { dificulty }(\text { high }) \\
\sqcup \text { dificulty }(\text { very high })\end{array}$ \\
\hline $\begin{array}{l}\text { The AVERAGE set of all learning objects that are in the portfolio } \\
\text { whose difficulty is medium is defined }\end{array}$ & AVERAGE $\equiv$ Artifact $\sqcap \exists$ describedBy.dificulty(medium) \\
\hline $\begin{array}{l}\text { The MINIMUM set of all the learning objects that are in the } \\
\text { portfolio whose difficulty is easy and very easy is defined. }\end{array}$ & $\begin{array}{c}\text { MINIMUM } \equiv \text { Artifact } \Pi \exists \text { describedBy. (dificulty (easy) } \\
\sqcup \text { dificulty(very easy) }\end{array}$ \\
\hline \multicolumn{2}{|c|}{ AGENT RULES } \\
\hline $\begin{array}{l}\text { IF answerGoForward(?p) THEN } \\
\text { Action= recommend HIGH. }\end{array}$ & $\begin{array}{l}\text { IF answerGoMinimun(?p) THEN } \\
\text { Action= recommend MINIMUN }\end{array}$ \\
\hline
\end{tabular}

instruments used in the proposed activity. In this sense, objective type questions (multiple options, simple options, true or false type question) generally used for simplicity of further correction, should be combined with other instruments such as essays to achieve student progress.

- This indicator, in short, will give professor the way to determine if an assessment is designed to determine higher levels of assimilation. The indicator, then, establishes the proportion of objective questions used in evaluations corresponding to a topic of the plan versus other types of questions identified as upperlevel questions corresponding to the same topic. Figure 3 shows that there is a taxonomy of terms that reflect the instruments available for an evaluation where different types of instruments (formal and semi-formal) are modeled. Instrument concept is used for this purpose. In the ontology, the term Assessment is related to the term Activity that models the questions of an evaluation, and Activity is linked to the term Instrument and its corresponding taxonomy. Instrument is related to the concept Classification [14] which is a metadata that distinguishes conceptual questions (metadata value is "conceptual") usually associated with objective instruments, from questions that are oriented to determine higher levels of learning assimilation ("deep" metadata value). Then, if the proportion of questions using activities with metadata classification "deep" is less than a value identified as desirable, it informs the teacher by recommending courses of action to modify the evaluation design.

In order to establish the type of activity or question available for an assessment, professor can take into account the keywords associated with the activity. Key words orientate the teacher to determine the corresponding level of assimilation [13]: Keywords such as define, enumerate, name or order in a statement are associated with the first level of assimilation. Instead, keywords such as classifying, expressing, locating, recognizing indicate that if the student answers the question correctly, he understands and interprets the information (level 2). Keywords as illustrate, demonstrate, employ are words that lead to determine the highest level of assimilation. These words are verbs that are associated with learning objectives. Therefore, in the ontology, the term Activity is related to the term Keyword through the relation hasKeyword representing the keywords associated with a given activity. The value of the metadata Classification will depend in part on this content.

That is, the content of the metadata classification associated with an activity or question available to be a part of an evaluation will depend on the instrument used and the keywords associated with it. In the ontology, the term Activity is related to the term DomainTopic expressing that an activity is also linked to a topic of the domain under study. Therefore, the teacher when proposing the inclusion of an evaluation in a portfolio must take into account that this evaluation should be designed taking into account the inclusion of activities that allow to establish clearly the level of assimilation of content by students in order to direct the learning process according to the individual progress favoring personalization.

\section{CONCLUSIONS}

This paper presents an ontology for the formalization of portfolios to determine the progress in learning process in a course. The ontology presented is part of an ontology network that models the different areas of interest to be considered in the domain of elearning. As a continuation of previous works, the developed ontology allows professor to make decisions about the learning path to be traversed by a student. The different stages in the learning path depend on the achievements of the students and are associated with his level of assimilation. In this sense, this proposal incorporates a series of concepts that allow educators to establish the level of assimilation of content by the students of a course based on information from their portfolios. This information is highly valuable for routing and directing instruction using materials suitable for this purpose. This way, the developed ontology becomes a powerful tool in order to personalize learning in higher education environments.

As part of the work, authors made progress on the definition 
of indicators to determine the assimilation of knowledge achieved by students. The assimilation of knowledge, or learning level, begins in the simple reproduction of a concept, going through the categorization of concepts until justifying a course of action and generating new ideas. For the calculation of the indicators, authors use information about instruments used to design assessments and about the type of activities proposed in each evaluation contained in a portfolio as well as the qualification obtained by the student.

The indicators were implemented through the definition of various terms and relations in the ontology and in logical rules that establish restrictions. In this sense, the progress of the work allows professors who are in charge of the planning of courses to obtain a series of recommendations to guide learning activities, select educational materials according to the possibilities of each student and, in this way, favor personalization.

Asfuturework, authorsproposethedefinitionofnewindicators that complement the pedagogical perspective on each student in particular and in learning process in general. Authors will also integrate the ontology to a software tool for portfolio management.

\section{REFERENCES}

[1] P.C. Sun, R.J. Tsai, G. Finger, Y.Y Chen and D. Yeh, "What drives a successful e-learning? An empirical investigation of the critical factors influencing learnir satisfaction", in Computers and education, Elsevier, 2008, pp.1183 - 1202.

[2] Romero, L., North, M., Gutiérrez, M., Caliusco, L.: Pedagogicallydriven ontology network for conceptualizing the e-learning assessment domain. Journal of educational technology and society IF: 1.34. 18(4) p.312-330. ISSN 1436-4522 (on-line), 1176-3647 (print). 2015. http://www.ifets.info/issues.php?show=current

[3] Romero, L., Gutierrez, M., \& Caliusco, M. L. (2012, June). Towards an ontology network for conceptualizing e-assessment domain. In Information Systems and Technologies (CISTI), 2012 7th Iberian Conference on (pp. 1-7).

[4] Romero, L., Gutierrez, M., \& Caliusco, M. L. (2013, June). A conceptualization of e-Assessment domain. In 2013 8th Iberian Conference on Information Systems and Technologies (CISTI) (pp. 1-6). IEEE. ISBN 978-989-96247-9-5.

[5] Romero, L., Gutiérrez, M., \& Caliusco, L. Towards Semantically Enriched E-learning Assessment: Ontology-Based Description of Learning Objects. In 2014 IEEE 14th International Conference on Advanced Learning Technologies (pp. 336-338).

[6] Romero, L., Gutierrez, M., \& Caliusco, M. L. (2015, June). Educational metadata ontology enrichment for e-assessment semantic description. Towards question and test interoperability and reuse. In 2015 10th Iberian Conference on Information Systems and Technologies (CISTI) (pp. 1-6).

[7] Romero, L., Gutierrez M., Caliusco M. Portfolio assessment to evaluate outcomes of learning in the e-learning environment. 11th Iberian Conference on Information Systems and Technologies (CISTI), Gran Canaria, 2016, pp. 1-7. doi: 10.1109/ CISTI.2016.7521406.

[8] Romero, L, Gutierrez, M., Caliusco, L. Semantic modeling of portfolio assessment in e-learning environment, Advances in Science, Technology and Engineering Systems Journal, vol. 2, no. 1, pp. 149-156 (2017).

[9] Romero, L., Gutierrez, Mi., Calliusco M. Conceptualizing the e-Learning Assessment Domain using an Ontology Network. IJIMAI.InternationalJournalofInteractiveMultimediaandArtificial Intelligence, Open Access. Special issue on Intelligent Systems and Applications.Number Vol I, número 6. P. 20-28. ISSN 1989-1660.

[10] Jaramillo Roldán, R. (2004): La calidad de la Educación: hacia un concepto de referencia. Revista Educación y Pedagogía ; $16 \quad$ (38): pp. 73-90.

[11] Nalda, F. N. (2002). La evaluación del aprendizaje y su influencia en el comportamiento estratégico del estudiante universitario. Contextos educativos: Revista de educación, (5), 141-156.
[12] Escorza, T. E. (2000). La evaluación y mejora de la enseñanza en la universidad: otra perspectiva. Revista de investigación Educativa, 18(2), 405-416.

[13] Leyva, L. M. L., Garrido, Y. P., Leyva, J. L. L., Varona, R. C., \& Rodríguez, R. R. (2007). Reflexiones sobre la evaluación de la calidad del aprendizaje en la práctica pedagógica en la escuela primaria. Revista Iberoamericana de Educación, 44(7), 3.

[14] IEEE LTSC, The IEEE Learning Technology Standards Committee, http://ieeeltsc.org/, last retrieved October 7th 2008.

[15] Chang, C. C., Tseng, K. H., \& Lou, S. J. (2012). A comparative analysis of the consistency and difference among teacherassessment, student self-assessment and peer-assessment in a Web-based portfolio assessment environment for high school students. Computers \& Education, 58(1), 303-320.

[16] Lorenzo, G., \&Ittelson, J. (2005). An overview of e-portfolios. Educause learning initiative, 1, 1-27.

[17] [17] Barrett, H. C., \& Garrett, N. (2009). Online personal learning environments: structuring electronic portfolios for lifelong and life-wide learning. On the Horizon, 17(2), 142-152.

[18] Mason, R., Pegler, C., \& Weller, M. (2004). Eportfolios: an assessment tool for online courses. British Journal of Educational Technology, 35(6), 717-727.

[19] Chang, C. C. (2001). A study on the evaluation and effectiveness analysis of web- based learning portfolio (WBLP). British Journal of Educational Technology, 32(4), 435-458.

[20] Bolivar C. (2011). Pruebas de rendimiento académico. Technical report. Programa interinstitucional doctorado en educación.

[21] EPAC ePortfolio-related Tools and Technologies. http://epac.pbworks.com/w/page/12559686/ Evolving \% 20 List \% C 2\%A 0 of\% C $2 \%$ A 0 e Portfoliorelated\%C2\%A0Tools [accedido, 16/02/2016.

[22] [Janssen, J., Berlanga, A. J., Vogten, H., Koper, R. (2008) Towards a learning path specification; International Journal of Continuing Engineering Education and Lifelong Learning, 18, 1 77-97. 